\title{
Unitary systems and wavelet sets
}

\author{
David R. Larson
}

\begin{abstract}
A wavelet is a special case of a vector in a separable Hilbert space that generates a basis under the action of a system of unitary operators defined in terms of translation and dilation operations. We will describe an operator-interpolation approach to wavelet theory using the local commutant of a unitary system. This is an application of the theory of operator algebras to wavelet theory. The concrete applications to wavelet theory include results obtained using specially constructed families of wavelet sets. The main section of this paper is section 5 , in which we introduce the interpolation map $\sigma$ induced by a pair of wavelet sets, and give an exposition of its properties and its utility in constructing new wavelets from old. The earlier sections build up to this, establishing terminology and giving examples. The main theoretical result is the Coefficient Criterion, which is described in Section 5.2.2, and which gives a matrix valued function criterion specificing precisely when a function with frequency support contained in the union of an interpolation family of wavelet sets is in fact a wavelet. This can be used to derive Meyer's famous class of wavelets using an interpolation pair of Shannon-type wavelet sets as a starting point. Section 5.3 contains a new result on interpolation pairs of wavelet sets: a proof that every pair of sets in the generalized Journe family of wavelet sets is an interpolation pair. We will discuss some results that are due to this speaker and his former and current students. And we finish in section 6 with a discussion of some open problems on wavelets and frame-wavelets.
\end{abstract}

Mathematics Subject Classification (2000). Primary 46L99; Secondary 42C15, $46 \mathrm{H} 25$.

Keywords. wavelet, wavelet set, unitary system, frame.

\section{Introduction}

A wavelet is a special case of a vector in a separable Hilbert space that generates a basis under the action of a collection, or "system", of unitary operators defined in

This research was supported by a grant from the NSF.. 
terms of translation and dilation operations. This approach to wavelet theory goes back, in particular, to earlier work of Goodman, Lee and Tang [10] in the context of multiresolution analysis. We will begin by describing the operator-interpolation approach to wavelet theory using the local commutant of a system that was worked out by the speaker and his collaborators a few years ago. This is really an abstract application of the theory of operator algebras, mainly von Neumann algebras, to wavelet theory. The concrete applications of operator-interpolation to wavelet theory include results obtained using specially constructed families of wavelet sets. In fact X. Dai and the speaker had originally developed our theory of wavelet sets [5] specifically to take advantage of their natural and elegant relationships with these wavelet unitary systems. We will also discuss some new results and open questions.

The main idea in operator-theoretic interpolation of wavelets (and frames) is that new wavelets can be obtained as linear combinations of known ones using coefficients which are operators (in fact, Fourier multipliers) in a certain class. Both the ideas and the essential computations extend naturally to more general unitary systems and wandering vectors. Many of the methods work for more involved systems that are important to applied harmonic analysis, such as Gabor and generalized Gabor systems, and various types of frame unitary systems.

\subsection{Terminology}

The set of all bounded linear operators on a Hilbert space $H$ will be denoted by $B(H)$. A bilateral shift $U$ on $H$ is a unitary operator $U$ for which there exists a closed linear subspace $E \subset H$ with the property that the family of subspaces $\left\{U^{n} E: n \in \mathbb{Z}\right\}$ are orthogonal and give a direct-sum decomposition of $H$. The subspace $E$ is called a complete wandering subspace for $U$, and the multiplicity of $U$ is defined to be the dimension of $E$. The strong operator topology on $B(H$ is the topology of pointwise convergence, and the weak operator topology is the weakest topology such that the vector functionals $\omega_{x, y}$ on $B(H)$ defined by $A \mapsto\langle A x, y\rangle$, $A \in B(H), x, y \in H$, are all continuous. An algebra of operators is a linear subspace of $B(H)$ which is closed under multiplication. An operator algebra is an algebra of operators which is norm-closed. A subset $\mathcal{S} \subset B(H)$ is called selfadjoint if whenever $A \in \mathcal{S}$ then also $A^{*} \in \mathcal{S}$. A $C^{*}$-algebra is a self-adjoint operator algebra. A von Neumann algebra is a $C^{*}$-algebra which is closed in the weak operator topology. For a unital operator algebra, it is well known that being closed in the weak operator topology is equivalent to being closed in the closed in the strong operator topology. The commutant of a set $\mathcal{S}$ of operators in $B(H)$ is the family of all operators in $B(H)$ that commute with every operator in $\mathcal{S}$. It is closed under addition and multiplication, so is an algebra. And it is clearly closed in both the weak operator topology and the strong operator topology. We use the standard prime notation for the commutant. So the commutant of a subset $\mathcal{S} \subset B(H)$ is denoted: $\mathcal{S}^{\prime}:=\{A \in B(H): A S=S A, S \in \mathcal{S}\}$. The commutant of a selfadjoint set of operators is clearly a von Neumann algebra. Moreover, by a famous theorem of Fuglede every operator which commutes with a normal operator $N$ also commutes 
with its adjoint $N^{*}$, and hence the commutant of any set of normal operators is also a von Neumann algebra. So, of particular relevance to this work, the commutant of any set of unitary operators is a von Neumann algebra.

1.1.1. Frames and Operators. A sequence of vectors $\left\{f_{j}\right\}$ in a separable Hilbert space $H$ is a frame (or frame sequence) if there exist constants $C_{1}, C_{2}>0$ such that

$$
C_{1}\|f\|_{2}^{2} \leq \sum_{j}\left|<f, f_{j}>\right|^{2} \leq C_{2}\|f\|_{2}^{2}
$$

for all $f \in H$. If $C_{1}=C_{2}$ the frame is called tight, and if $C_{1}=C_{2}=1,\left\{f_{j}\right\}$ is called a $P$ arseval frame. (The term normalized tight has also been used for this (cf [14]). A vector $\xi$ is called a $f$ rame vector for a unitary system $\mathcal{U}$ if the set of vectors $\mathcal{U} \xi$ is a frame for $H$.

A Riesz basis for a Hilbert space is a bounded unconditional basis. Frames sequences are generalizations of Riesz bases. A number of the basic aspects of a geometric, or operator-theoretic, approach to discrete frame theory on Hilbert space arises from the fact that a frame sequence is simply an "inner" direct summand of a Riesz basis. The basic principle is that a Hilbert space frame sequence can be dilated to a Riesz basis for larger Hilbert space. We call this the Frame Dilation Theorem. In other words, for a given frame sequence there is a larger Hilbert space and a Riesz basis for the larger space such that the orthogonal projection from the larger space onto the smaller space compresses the Riesz basis to the frame sequence. We proved this at the beginning of [14], and used it to prove the other results [14], and subsequently to prove some applications to Hilbert $\mathrm{C}^{*}$ module theory jointly with M. Frank. We proved it first for Parseval frames, and then for general frames. (We remark that this type of dilation result for frames was also independently known and used independently by several others in different contexts.)

It is interesting to note that the Parseval frame case of the Frame Dilation Theorem can be derived easily from the purely atomic case of a well known theorem of Naimark on projection valued measures. We thank Chandler Davis for pointing this out to us at the Canadian Operator Algebras Symposium in 1999. We (Han and I) basically proved this special case of Naimark's theorem implicitely in the first section of [14] without recognizing it was a special case of Naimark's theorem, and then we proved the appropriate generalization we needed for general (nontight) frames. Naimark's Dilation Theorem basically states that a suitable positive operator valued measure on a Hilbert space dilates to a projection valued measure on a larger Hilbert space. That is, there is a projection valued measure [PVM] on a larger Hilbert space such that the orthogonal projection from the larger space unto the smaller space compresses the $[\mathrm{PVM}]$ to the $[\mathrm{POVM}]$. In the discrete (i.e. purely atomic measure) case, it can be interpreted as stating that a suitable sequence of positive operators dilates to a sequence of projections. The dilation theorem for a Parseval frame follows easily from Naimark's Theorem applied to the [POVM] obtained by replacing each vector $x_{i}$ in the frame sequence with the elementary 
tensor operator $x_{i} \otimes x_{i}$, obtaining the atoms for a [POVM] defined on all subsets of the index set for the frame. The dilation theorem for a general (non-tight) frame does not seem to follow directly from Naimark's theorem - but it may follow from a generalization of it. We remark that some other generalizations of the frame dilation theorem have been recently worked out, notably by W. Czaja.

1.1.2. Unitary Systems, Wandering Vectors, and Frame Vectors. We define a unitary system to be simply a countable collection of unitary operators $\mathcal{U}$ acting on a Hilbert space $H$ which contains the identity operator. The interesting unitary systems all have additional structural properties of various types. (For instance, wavelet systems and Gabor systems are both "ordered products" of two abelian groups: the dilation and translation groups in the wavelet case, and the modulation and translation groups in the Gabor case.) We will say that a vector $\psi \in H$ is wandering for $\mathcal{U}$ if the set

$$
\mathcal{U} \psi:=\{U \psi: U \in \mathcal{U}\}
$$

is an orthonormal set, and we will call $\psi$ a complete wandering vector for $\mathcal{U}$ if $\mathcal{U} \psi$ spans $H$. This (abstract) point of view can be useful. Write $\mathcal{W}(\mathcal{U})$ for the set of complete wandering vectors for $\mathcal{U}$. Further, a Riesz vector for $\mathcal{U}$ is a vector $\psi$ such that $\mathcal{U} \psi$ is a Riesz basis for $H$ (indexed by the elements of $\mathcal{U}$ ), and a frame vector is a vector $\psi$ such that $\mathcal{U} \psi$ is a frame sequence for $H$ (again using $\mathcal{U}$ as its index set), and we adopt similar terminology for Parseval frame vectors and Bessel vectors. We use $\mathcal{R} W(\mathcal{U}), \mathcal{F}(\mathcal{U}), \mathcal{P} F(\mathcal{U}), \mathcal{B}(\mathcal{U})$ to denote, respectively, the sets of Riesz vectors, frame vectors, Parseval frame vectors, and Bessel vectors for $\mathcal{U}$.

One of the main tools in this work is the local commutant of a system of unitary operators (see section 3.2). This is a natural generalization of the commutant of the system, and like the commutant it is a linear space of operators which is closed in the weak and the strong operator topologies, but unlike the commutant it is usually not selfadjoint, and is usually not closed under multiplication. It contains the commutant of the system, but can be much larger than the commutant. The local commutant of a wavelet unitary system captures all the information about the wavelet system in an essential way, and this gives the flavor of our approach to the subject.

1.1.3. Normalizers. If $U$ is a unitary operator and $\mathcal{A}$ is an operator algebra, then $U$ is said to normalize $\mathcal{A}$ if $U^{\star} \cdot \mathcal{A} \cdot U=\mathcal{A}$. In the most interesting cases of operatortheoretic interpolation: that is, for those cases that yield the strongest structural results, the relevant unitaries in the local commutant of the system normalize the commutant of the system.

\subsection{Acknowledgement}

This article was written in response to an invitation by the organizers of the 4th International Conference on Wavelet Analysis and its Applications, Macau, China, December 2005 [WAA2005] to be a keynote speaker. We thank the organizers for their kind invitation to present these notes. 


\section{Wavelets}

For simplicity of presentation, much of the work in this article will deal with onedimensional wavelets, and in particular, the dyadic case. The other cases: nondyadic wavelets and wavelets in higher dimensions, are at least notationally more complicated.

\subsection{One Dimension}

A dyadic orthonormal wavelet in one dimension is a unit vector $\psi \in L^{2}(\mathbb{R}, \mu)$, with $\mu$ Lebesgue measure, with the property that the set

$$
\left\{2^{\frac{n}{2}} \psi\left(2^{n} t-l\right): n, l \in \mathbb{Z}\right\}
$$

of all integral translates of $\psi$ followed by dilations by arbitrary integral powers of 2 , is an orthonormal basis for $L^{2}(\mathbb{R}, \mu)$. The term dyadic refers to the dilation factor " 2 ". The term mother wavelet is also used in the literature for $\psi$. Then the functions

$$
\psi_{n, l}:=2^{\frac{n}{2}} \psi\left(2^{n} t-l\right)
$$

are called elements of the wavelet basis generated by the "mother". The functions $\psi_{n, l}$ will not themselves be mother wavelets unless $n=0$.

Let $T$ and $D$ be the translation (by 1) and dilation (by 2) unitary operators in $B\left(L^{2}(\mathbb{R})\right.$ given by $(T f)(t)=f(t-1)$ and $(D f)(t)=\sqrt{2} f(2 t)$. Then

$$
2^{\frac{n}{2}} \psi\left(2^{n} t-l\right)=\left(D^{n} T^{l} \psi\right)(t)
$$

for all $n, l \in \mathbb{Z}$. Operator-theoretically, the operators $T, D$ are bilateral shifts of infinite multiplicity. It is obvious that $L^{2}([0,1])$, considered as a subspace of $L^{2}(\mathbb{R})$, is a complete wandering subspace for $T$, and that $L^{2}([-2,-1] \cup[1,2])$ is a complete wandering subspace for $D$.

Let $\mathcal{U}_{D, T}$ be the unitary system defined by

$$
\mathcal{U}_{D, T}=\left\{D^{n} T^{l}: n, l \in \mathbb{Z}\right\}
$$

where $D$ and $T$ are the operators defined above. Then $\psi$ is a dyadic orthonormal wavelet if and only if $\psi$ is a complete wandering vector for the unitary system $\mathcal{U}_{D, T}$. This was our original motivation for developing the abstract unitary system theory. Write

$$
\mathcal{W}(D, T):=\mathcal{W}\left(\mathcal{U}_{D, T}\right)
$$

to denote the set of all dyadic orthonormal wavelets in one dimension.

An abstract interpretation is that, since $D$ is a bilateral shift it has (many) complete wandering subspaces, and a wavelet for the system is a vector $\psi$ whose translation space (that is, the closed linear span of $\left\{T^{k}: k \in \mathbb{Z}\right\}$ is a complete wandering subspace for $D$. Hence $\psi$ must generate an orthonormal basis for the entire Hilbert space under the action of the unitary system.

In one dimension, there are non-dyadic orthonormal wavelets: i.e. wavelets for all possible dilation factors besides 2 (the dyadic case). We said "possible", because the scales $\{0,1,-1\}$ are excluded as scales because the dilation operators they would introduce are not bilateral shifts. All other real numbers for scales 
yield wavelet theories. In [5, Example $4.5(\mathrm{x})$ ] a family of examples is given of three-interval wavelet sets (and hence wavelets) for all scales $d \geq 2$, and it was noted there that such a family also exists for dilation factors $1<d \leq 2$. There is some recent (yet unpublished) work that has been done, by REU students and mentors, building on this, classifying finite-interval wavelet sets for all possible real (positive and negative scale factors). I will mention this work, in passing, in my talk.

\subsection{N-Dimensions}

2.2.1. Expansive Dilations. Let $1 \leq m<\infty$, and let $A$ be an $n \times n$ real matrix which is expansive (equivalently, all (complex) eigenvalues have modulus $>1$ ). By a dilation - A regular-translation orthonormal wavelet we mean a function $\psi \in L^{2}\left(\mathbb{R}^{n}\right)$ such that

$$
\left\{|\operatorname{det}(A)|^{\frac{n}{2}} \psi\left(A^{n} t-\left(l_{1}, l_{2}, \ldots, l_{n}\right)^{t}\right): n, l \in \mathbb{Z}\right\}
$$

where $t=\left(t_{1}, \ldots, t_{n}\right)^{t}$, is an orthonormal basis for $L^{2}\left(\mathbb{R}^{n} ; m\right)$. (Here $m$ is product Lebesgue measure, and the superscript " $t$ " means transpose.)

If $A \in M_{n}(\mathbb{R})$ is invertible (so in particular if $A$ is expansive), then it is very easy to verify that the operator defined by

$$
\left(D_{A} f\right)(t)=|\operatorname{det} A|^{\frac{1}{2}} f(A t)
$$

for $f \in L^{2}\left(\mathbb{R}^{n}\right), t \in \mathbb{R}^{n}$, is unitary. For $1 \leq i \leq n$, let $T_{i}$ be the unitary operator determined by translation by 1 in the $i^{\text {th }}$ coordinate direction. The set (5) above is then

$$
\left\{D_{A}^{k} T_{1}^{l_{1}} \cdots T_{n}^{l_{n}} \psi: k, l_{i} \in \mathbb{Z}\right\}
$$

If the dilation matrix $A$ is expansive, but the translations are along some oblique lattice, then there is an invertible real $n \times n$ matrix $T$ such that conjugation with $D_{T}$ takes the entire wavelet system to a regular-translation expansive-dilation matrix. This is easily worked out, and was shown in detail in [18] in the context of working out a complete theory of unitary equivalence of wavelet systems. Hence the wavelet theories are equivalent.

2.2.2. Non-Expansive Dilations. Much work has been accomplished concerning the existence of wavelets for dilation matrices $A$ which are not expansive. Some of the original work was accomplished in the Ph.D. theses of Q. Gu and D. Speegle, when they were together finishing up at Texas A\&M. Some significant additional work was accomplished by Speegle and also by others. In [18], with Ionascu and Pearcy we proved that if an $n x n$ real invertible matrix $A$ is not similar (in the nxn complex matrices) to a unitary matrix, then the corresponding dilation operator $D_{A}$ is in fact a bilateral shift of infinite multiplicity. If a dilation matrix were to admit any type of wavelet (or frame-wavelet) theory, then it is well-known that a necessary condition would be that the corresponding dilation operator would have to be a bilateral shift of infinite multiplicity. I am happy to report that in very recent work [23], with E. Schulz, D. Speegle, and K. Taylor, we have succeeded in showing that this minimal condition is in fact sufficient: such a matrix, with 
regular translation lattice, admits a (perhaps infinite) tuple of functions, which collectively generates a frame-wavelet under the action of this unitary system.

\section{More General Unitary Systems}

\subsection{Some Restrictions}

We note that most unitary systems $\mathcal{U}$ do not have complete wandering vectors. For $\mathcal{W}(\mathcal{U})$ to be nonempty, the set $\mathcal{U}$ must be very special. It must be countable if it acts separably (i.e. on a separable Hilbert space), and it must be discrete in the strong operator topology because if $U, V \in \mathcal{U}$ and if $x$ is a wandering vector for $\mathcal{U}$ then

$$
\|U-V\| \geq\|U x-V x\|=\sqrt{2}
$$

Certain other properties are forced on $\mathcal{U}$ by the presence of a wandering vector. (Or indeed, by the nontriviality of any of the sets $\mathcal{W}(\mathcal{U}), \mathcal{R} \mathcal{W}(\mathcal{U}), \mathcal{F}(\mathcal{U}), \mathcal{P} \mathcal{F}(\mathcal{U})$, $\mathcal{B}(\mathcal{U})$.) One purpose of [5] was to investigate such properties. Indeed, it was a matter of some surprise to us to discover that such a theory is viable even in some considerable generality. For perspective, it is useful to note that while $\mathcal{U}_{D, T}$ has complete wandering vectors, the reversed system

$$
\mathcal{U}_{T, D}=\left\{T^{l} D^{n}: n, l \in \mathbb{Z}\right\}
$$

fails to have a complete wandering vector. (A proof of this was given in the introduction to [5].)

\subsection{The Local Commutant}

3.2.1. A Special Case: The System $\mathcal{U}_{D, T}$. Computational aspects of operator theory can be introduced into the wavelet framework in an elementary way. Here is the way we originally did it: Fix a wavelet $\psi$ and consider the set of all operators $A \in B\left(L^{2}(\mathbb{R})\right)$ which commute with the action of dilation and translation on $\psi$. That is, require

$$
(A \psi)\left(2^{n} t-l\right)=A\left(\psi\left(2^{n} t-l\right)\right)
$$

or equivalently

$$
D^{n} T^{l} A \psi=A D^{n} T^{l} \psi
$$

for all $n, l \in \mathbb{Z}$. Call this the local commutant of the wavelet system $\mathcal{U}_{D, T}$ at the vector $\psi$. (In our first preliminary writings and talks we called it the point commutant of the system.) Formally, the local commutant of the dyadic wavelet system on $L^{2}(\mathbb{R})$ is:

$$
\mathcal{C}_{\psi}\left(\mathcal{U}_{D, T}\right):=\left\{A \in B\left(L^{2}(\mathbb{R})\right):\left(A D^{n} T^{l}-D^{n} T^{l} A\right) \psi=0, \forall n, l \in \mathbb{Z}\right\}
$$

This is a linear subspace of $B(H)$ which is closed in the strong operator topology, and in the weak operator topology, and it clearly contains the commutant of $\{D, T\}$.

A motivating example is that if $\eta$ is any other wavelet, let $V:=V_{\psi}^{\eta}$ be the unitary (we call it the interpolation unitary) that takes the basis $\psi_{n, l}$ to the basis 
$\eta_{n, l}$. That is, $V \psi_{n, l}=\eta_{n, l}$ for all $n, l \in \mathbb{Z}$. Then $\eta=V \psi$, so $V D^{n} T^{l} \psi=D^{n} T^{l} V \psi$ hence $V \in \mathcal{C}_{\psi}\left(\mathcal{U}_{D, T}\right)$.

In the case of a pair of complete wandering vectors $\psi, \eta$ for a general unitary system $\mathcal{U}$, we will use the same notation $V_{\psi}^{\eta}$ for the unitary that takes the vector $U \psi$ to $U \eta$ for all $U \in \mathcal{U}$.

This simple-minded idea is reversible, so for every unitary $V$ in $\mathcal{C}_{\psi}\left(\mathcal{U}_{D, T}\right)$ the vector $V \psi$ is a wavelet. This correspondence between unitaries in $\mathcal{C}_{\psi}(D, T)$ and dyadic orthonormal wavelets is one-to-one and onto (see Proposition 3.1). This turns out to be useful, because it leads to some new formulas relating to decomposition and factorization results for wavelets, making use of the linear and multiplicative properties of $\mathcal{C}_{\psi}(D, T)$.

It turns out (a proof is required) that the entire local commutant of the system $\mathcal{U}_{D, T}$ at a wavelet $\psi$ is not closed under multiplication, but it also turns out (also via a proof) that for most (and perhaps all) wavelets $\psi$ the local commutant at $\psi$ contains many noncommutative operator algebras (in fact von Neumann algebras) as subsets, and their unitary groups parameterize norm-arcwise-connected families of wavelets. Moreover, $\mathcal{C}_{\psi}(D, T)$ is closed under left multiplication by the commutant $\{D, T\}^{\prime}$, which turns out to be an abelian nonatomic von Neumann algebra. The fact that $\mathcal{C}_{\psi}(D, T)$ is a left module under $\{D, T\}^{\prime}$ leads to a method of obtaining new wavelets from old, and of obtaining connectedness results for wavelets, which we called operator-theoretic interpolation of wavelets in [5], (or simply operator-interpolation).

3.2.2. General Systems. More generally, let $\mathcal{S} \subset B(H)$ be a set of operators, where $H$ is a separable Hilbert space, and let $x \in H$ be a nonzero vector, and formally define the local commutant of $\mathcal{S}$ at $x$ by

$$
\mathcal{C}_{x}(\mathcal{S}):=\{A \in B(H):(A S-S A) x=0, S \in \mathcal{S}\}
$$

As in the wavelet case, this is a weakly and strongly closed linear subspace of $B(H)$ which contains the commutant $\mathcal{S}^{\prime}$ of $\mathcal{S}$. If $x$ is cyclic for $\mathcal{S}$ in the sense that $\operatorname{span}(\mathcal{S} x)$ is dense in $H$, then $x$ separates $\mathcal{C}_{x}(\mathcal{S})$ in the sense that for $S \in \mathcal{C}_{x}(\mathcal{S})$, we have $S x=0$ iff $x=0$. Indeed, if $A \in \mathcal{C}_{x}(\mathcal{S})$ and if $A x=0$, then for any $S \in \mathcal{S}$ we have $A S x=S A x=0$, so $A \mathcal{S} x=0$, and hence $A=0$.

If $A \in \mathcal{C}_{x}(\mathcal{S})$ and $B \in \mathcal{S}^{\prime}$, let $C=B A$. Then for all $S \in \mathcal{S}$,

$$
(C S-S C) x=B(A S) x-(S B) A x=B(S A) x-(B S) A x=0
$$

because $A S x=S A x$ since $A \in \mathcal{C}_{x}(\mathcal{S})$, and $S B=B S$ since $B \in \mathcal{S}^{\prime}$. Hence $\mathcal{C}_{x}(\mathcal{S})$ is closed under left multiplication by operators in $\mathcal{S}^{\prime}$. That is, $\mathcal{C}_{x}(\mathcal{S}$ is a left module over $\mathcal{S}^{\prime}$.

It is interesting that, if in addition $\mathcal{S}$ is a multiplicative semigroup, then in fact $\mathcal{C}_{x}(\mathcal{S})$ is identical with the commutant $\mathcal{S}^{\prime}$ so in this case the commutant is not a new structure. To see this, suppose $A \in \mathcal{C}_{x}(\mathcal{S})$. Then for each $S, T \in \mathcal{S}$ we have $S T \in \mathcal{S}$, and so

$$
A S(T x)=(S T) A x=S(A T x)=(S) T x
$$


So since $T \in \mathcal{S}$ was arbitrary and $\operatorname{span}(\mathcal{S} x)=H$, it follows that $A S=S A$.

Proposition 3.1. If $\mathcal{U}$ is any unitary system for which $\mathcal{W}(\mathcal{U}) \neq \emptyset$, then for any $\psi \in \mathcal{W}(\mathcal{U})$

$$
\mathcal{W}(\mathcal{U})=\left\{U \psi: U \text { is a unitary operator in } \mathcal{C}_{\psi}(\mathcal{U})\right\}
$$

and the correspondence $U \rightarrow U \psi$ is one-to-one.

Proposition 3.2. Let $\mathcal{U}$ be a unitary system on a Hilbert space $H$. If $\psi$ is a complete wandering vector for $\mathcal{U}$, then:

(i) $\mathcal{R} \mathcal{W}(\mathcal{U})=\left\{A \psi: A\right.$ is an operator in $\mathcal{C}_{\psi}(\mathcal{U})$ that is invertible in $\left.B(H)\right\}$;

(ii) $\mathcal{F}(\mathcal{U})=\left\{A \psi: A\right.$ is an operator in $\mathcal{C}_{\psi}(\mathcal{U})$ that is surjective $\}$;

(ii) $\mathcal{P} \mathcal{F}(\mathcal{U})=\left\{A \psi: A\right.$ is an operator in $\mathcal{C}_{\psi}(\mathcal{U})$ that is a co-isometry $\}$;

(ii) $\mathcal{B}(\mathcal{U})=\left\{A \psi: A\right.$ is an operator in $\left.\mathcal{C}_{\psi}(\mathcal{U})\right\}$

\subsection{Operator-Theoretic Interpolation}

Now suppose $\mathcal{U}$ is a unitary system, such as $\mathcal{U}_{D, T}$, and suppose $\left\{\psi_{1}, \psi_{2}, \ldots, \psi_{m}\right\} \subset$ $\mathcal{W}(\mathcal{U})$. (In the case of $\mathcal{U}_{D, T}$, this means that $\left(\psi_{1}, \psi_{2}, \ldots, \psi_{n}\right)$ is an n-tuple of wavelets.

Let $\left(A_{1}, A_{2}, \ldots, A_{n}\right)$ be an n-tuple of operators in the commutant $\mathcal{U}^{\prime}$ of $\mathcal{U}$, and let $\eta$ be the vector

$$
\eta:=A_{1} \psi_{1}+A_{2} \psi_{2}+\cdots+A_{n} \psi_{n} .
$$

Then

$$
\begin{aligned}
\eta & =A_{1} \psi_{1}+A_{2} V_{\psi_{1}}^{\psi_{2}} \psi_{1}+\ldots A_{n} V_{\psi_{1}}^{\psi_{n}} \psi_{1} \\
& =\left(A_{1}+A_{2} V_{\psi_{1}}^{\psi_{2}}+\cdots+A_{n} V_{\psi_{1}}^{\psi_{n}}\right) \psi_{1} .
\end{aligned}
$$

We say that $\eta$ is obtained by operator interpolation from $\left\{\psi_{1}, \psi_{2}, \ldots, \psi_{m}\right\}$. Since $\mathcal{C}_{\psi_{1}}(\mathcal{U})$ is a left $\mathcal{U}^{\prime}$ - module, it follows that the operator

$$
A:=A_{1}+A_{2} V_{\psi_{1}}^{\psi_{2}}+\ldots A_{n} V_{\psi_{1}}^{\psi_{n}}
$$

is an element of $\mathcal{C}_{\psi_{1}}(\mathcal{U})$. Moreover, if $B$ is another element of $\mathcal{C}_{\psi_{1}}(\mathcal{U})$ such that $\eta=B \psi_{1}$, then $A-B \in \mathcal{C}_{\psi_{1}}(\mathcal{U})$ and $(A-B) \psi_{1}=0$. So since $\psi_{1}$ separates $\mathcal{C}_{\psi_{1}}(\mathcal{U})$ it follows that $A=B$. Thus $A$ is the unique element of $\mathcal{C}_{\psi_{1}}(\mathcal{U})$ that takes $\psi_{1}$ to $\eta$. Let $\mathcal{S}_{\psi_{1}, \ldots, \psi_{n}}$ be the family of all finite sums of the form

$$
\sum_{i=0}^{n} A_{i} V_{\psi_{1}}^{\psi_{i}} .
$$

This is the left module of $\mathcal{U}^{\prime}$ generated by $\left\{I, V_{\psi_{1}}^{\psi_{2}}, \ldots, V_{\psi_{1}}^{\psi_{n}}\right\}$. It is the $\mathcal{U}^{\prime}$-linear span of $\left\{I, V_{\psi_{1}}^{\psi_{2}}, \ldots, V_{\psi_{1}}^{\psi_{n}}\right\}$.

Let

$$
\mathcal{M}_{\psi_{1}, \ldots, \psi_{n}}:=\left(\mathcal{S}_{\psi_{1}, \ldots, \psi_{n}}\right) \psi_{1}
$$


So

$$
\mathcal{M}_{\psi_{1}, \ldots, \psi_{n}}=\left\{\sum_{i=0}^{n} A_{i} \psi_{i}: A_{i} \in \mathcal{U}^{\prime}\right\} .
$$

We call this the interpolation space for $\mathcal{U}$ generated by $\left(\psi_{1}, \ldots, \psi_{n}\right)$. From the above discussion, it follows that for every vector $\eta \in \mathcal{M}_{\psi_{1}, \psi_{2}, \ldots, \psi_{n}}$ there exists a unique operator $A \in \mathcal{C}_{\psi_{1}}(\mathcal{U})$ such that $\eta=A \psi_{1}$, and moreover this $A$ is an element of $\mathcal{S}_{\psi_{1}, \ldots, \psi_{n}}$.

3.3.1. Normalizing the Commutant. In certain essential cases (and we are not sure how general this type of case is) one can prove that an interpolation unitary $V_{\psi}^{\eta}$ normalizes the commutant $\mathcal{U}^{\prime}$ of the system in the sense that $V_{\eta}^{\psi} \mathcal{U}^{\prime} V_{\psi}^{\eta}=\mathcal{U}^{\prime}$. (Here, it is easily seen that $\left(V_{\psi}^{\eta}\right)^{*}=V_{\eta}^{\psi}$.) Write $V:=V_{\psi}^{\eta}$. If $V$ normalizes $\mathcal{U}^{\prime}$, then the algebra, before norm closure, generated by $\mathcal{U}^{\prime}$ and $V$ is the set of all finite sums (trig polynomials) of the form $\sum A_{n} V^{n}$, with coefficients $A_{n} \in \mathcal{U}^{\prime}, n \in \mathbb{Z}$. The closure in the strong operator topology is a von Neumann algebra. Now suppose further that every power of $V$ is contained in $\mathcal{C}_{\psi}(\mathcal{U})$. This occurs only in special cases, yet it occurs frequently enough to yield some general methods. Then since $\mathcal{C}_{\psi}(\mathcal{U})$ is a SOT-closed linear subspace which is closed under left multiplication by $\mathcal{U}^{\prime}$, this von Neumann algebra is contained in $\mathcal{C}_{\psi}(\mathcal{U})$, so its unitary group parameterizes a norm-path-connected subset of $\mathcal{W}(\mathcal{U})$ that contains $\psi$ and $\eta$ via the correspondence $U \rightarrow U \psi$.

In the special case of wavelets, this is the basis for the work that Dai and I did in [5, Chapter 5] on operator-theoretic interpolation of wavelets. In fact, we specialized there and reserved the term operator-theoretic interpolation to refer explicitly to the case when the interpolation unitaries normalize the commutant. In some subsequent work, we loosened this restriction yielding our more general definition given in this article, because there are cases of interest in which we weren't able to prove normalization. However, it turns out that if $\psi$ and $\eta$ are $s$-elementary wavelets (see section 4.4), then indeed $V_{\psi}^{\eta}$ normalizes $\{D, T\}^{\prime}$. (See Proposition 5.3.) Moreover, $V_{\psi}^{\eta}$ has a very special form: after conjugating with the Fourier transform, it is a composition operator with a symbol $\sigma$ that is a natural and very computable measure-preserving transformation of $\mathbb{R}$. In fact, it is precisely this special form for $V_{\psi}^{\eta}$ that allows us to make the computation that it normalizes $\{D, T\}^{\prime}$. On the other hand, we know of no pair $(\psi, \eta)$ of wavelets for which $V_{\psi}^{\eta}$ fails to normalize $\{D, T\}^{\prime}$. The difficulty is simply that in general it is very hard to do the computations. This is stated as Problem 2 in the final section on Open Problems.

In the wavelet case $\mathcal{U}_{D, T}$, if $\psi \in \mathcal{W}(D, T)$ then it turns out that $\mathcal{C}_{\psi}\left(\mathcal{U}_{D, T}\right)$ is in fact much larger than $\left(\mathcal{U}_{D, T}\right)^{\prime}=\{D, T\}^{\prime}$, underscoring the fact that $\mathcal{U}_{D, T}$ is NOT a group. In particular, $\{D, T\}^{\prime}$ is abelian while $\mathcal{C}_{\psi}(\mathcal{D}, \mathcal{T})$ is nonabelian for every wavelet $\psi$. (The proof of these facts are contained in [5].) 
3.3.2. Interpolation Pairs of Wandering Vectors. In some cases where a pair $\psi, \eta$ of vectors in $\mathcal{W}(\mathcal{U})$ are given it turns out that the unitary $V$ in $\mathcal{C}_{\psi}(\mathcal{U})$ with $V \psi=$ $\eta$ happens to be a symmetry (i.e. $V^{2}=I$ ). Such pairs are called interpolation pairs of wandering vectors, and in the case where $\mathcal{U}$ is a wavelet system, they are called interpolation pairs of wavelets. Interpolation pairs are more prevalent in the theory, and in particular the wavelet theory, than one might expect. In this case (and in more complex generalizations of this) certain linear combinations of complete wandering vectors are themselves complete wandering vectors - not simply complete Riesz vectors.

Proposition 3.3. Let $\mathcal{U}$ be a unitary system, let $\psi, \eta \in \mathcal{W}(\mathcal{U})$, and let $V$ be the unique operator in $\mathcal{C}_{\psi}(\mathcal{U})$ with $V \psi=\eta$. Suppose

$$
V^{2}=I
$$

Then

$$
\cos \alpha \cdot \psi+i \sin \alpha \cdot \eta \in \mathcal{W}(\mathcal{U})
$$

for all $0 \leq \alpha \leq 2 \pi$.

The above result can be thought of as the prototype of our operator-theoretic interpolation results. It is the second most elementary case. (The most elementary case is described in the context of the exposition of Problem 4 in the final section.) More generally, the scalar $\alpha$ in Proposition 3.3 can be replaced with an appropriate self-adjoint operator in the commutant of $\mathcal{U}$. In the wavelet case, after conjugating with the Fourier transform, which is a unitary operator, this means that $\alpha$ can be replaced with a wide class of nonnegative dilation-periodic (see definition below) bounded measurable functions on $\mathbb{R}$.

\section{Wavelet Sets}

Wavelet sets belong to the theory of wavelets via the Fourier Transform. We will do most of this section in a tutorial-style, to make the concepts more accessible to students and colleagues who are not already familiar with them.

\subsection{Fourier Transform}

We will use the following form of the Fourier-Plancherel transform $\mathcal{F}$ on $\mathcal{H}=$ $L^{2}(\mathbb{R})$, which is a form that is normalized so it is a unitary transformation, a property that is desirable for our treatment.

If $f, g \in L^{1}(\mathbb{R}) \cap L^{2}(\mathbb{R})$ then

$$
(\mathcal{F} f)(s):=\frac{1}{\sqrt{2 \pi}} \int_{\mathbb{R}} e^{-i s t} f(t) d t:=\hat{f}(s),
$$

and

$$
\left(\mathcal{F}^{-1} g\right)(t)=\frac{1}{\sqrt{2 \pi}} \int_{\mathbb{R}} e^{i s t} g(s) d s .
$$


We have

$$
\left(\mathcal{F} T_{\alpha} f\right)(s)=\frac{1}{\sqrt{2 \pi}} \int_{\mathbb{R}} e^{-i s t} f(t-\alpha) d t=e^{-i s \alpha}(\mathcal{F} f)(s) .
$$

So $\mathcal{F} T_{\alpha} \mathcal{F}^{-1} g=e^{-i s \alpha} g$. For $A \in \mathcal{B}(\mathcal{H})$ let $\hat{A}$ denote $\mathcal{F} A \mathcal{F}^{-1}$. Thus

$$
\widehat{T}_{\alpha}=M_{e^{-i \alpha s}},
$$

where for $h \in L^{\infty}$ we use $M_{h}$ to denote the multiplication operator $f \rightarrow h f$. Since $\left\{M_{e^{-i \alpha s}}: \quad \alpha \in \mathbb{R}\right\}$ generates the m.a.s.a. $\mathcal{D}(\mathbb{R}):=\left\{M_{h}: h \in L^{\infty}(\mathbb{R})\right\}$ as a von Neumann algebra, we have

$$
\mathcal{F} \mathcal{A}_{T} \mathcal{F}^{-1}=\mathcal{D}(\mathbb{R})
$$

Similarly,

$$
\begin{aligned}
\left(\mathcal{F} D^{n} f\right)(s) & =\frac{1}{\sqrt{2 \pi}} \int_{\mathbb{R}} e^{-i s t}(\sqrt{2})^{n} f\left(2^{n} t\right) d t \\
& =(\sqrt{2})^{-n} \cdot \frac{1}{\sqrt{2 \pi}} \int_{\mathbb{R}} e^{-i 2^{-n} s t} f(t) d t \\
& =(\sqrt{2})^{-2}(\mathcal{F} f)\left(2^{2^{-n}} s\right)=\left(D^{-n} \mathcal{F} f\right)(s) .
\end{aligned}
$$

So $\widehat{D}^{n}=D^{-n}=D^{* n}$. Therefore,

$$
\widehat{D}=D^{-1}=D^{*}
$$

If $f$ is an $L^{2}(\mathbb{R})$ function, as usual we write $\widehat{f}(s)=(\mathcal{F}(f))(s)$. If $\rho(s)$ is a real-valued function such that $\widehat{f}(s)=e^{i \rho(s)}|\widehat{f}(s)|$, we call $\rho(s)$ the phase of $f$. The phase is well defined a.e. modulo $2 \pi$-translation.

\subsection{The Commutant of $\{D, T\}$}

We have $\mathcal{F}\{D, T\}^{\prime} \mathcal{F}^{-1}=\{\widehat{D}, \widehat{T}\}^{\prime}$. It turns out that $\{\widehat{D}, \widehat{T}\}^{\prime}$ has an elementary characterization in terms of Fourier multipliers:

\section{Theorem 4.1.}

$$
\{\widehat{D}, \widehat{T}\}^{\prime}=\left\{M_{h}: h \in L^{\infty}(\mathbb{R}) \text { and } h(s)=h(2 s) \text { a.e. }\right\} .
$$

Proof. Since $\widehat{D}=D^{*}$ and $D$ is unitary, it is clear that $M_{h} \in\{\widehat{D}, \widehat{T}\}^{\prime}$ if and only if $M_{h}$ commutes with $D$. So let $g \in L^{2}(\mathbb{R})$ be arbitrary. Then (a.e.) we have

$$
\begin{aligned}
& \left(M_{h} D g\right)(s)=h(s)(\sqrt{2} g(2 s)), \quad \text { and } \\
& \left(D M_{h} g\right)(s)=D(h(s) g(s))=\sqrt{h}(2 s) g(2 s) .
\end{aligned}
$$

Since these must be equal a.e. for arbitrary $g$, we must have $h(s)=h(2 s)$ a.e.

Now let $E=[-2,-1) \cup[1,2)$, and for $n \in \mathbb{Z}$ let $E_{n}=\left\{2^{n} x: x \in E\right\}$. Observe that the sets $E_{n}$ are disjoint and have union $\mathbb{R} \backslash\{0\}$. So if $g$ is any uniformly bounded function on $E$, then $g$ extends uniquely (a.e.) to a function $\tilde{g} \in L^{\infty}(\mathbb{R})$ satisfying

$$
\tilde{g}(s)=\tilde{g}(2 s), \quad s \in \mathbb{R},
$$


by setting

$$
\tilde{g}\left(2^{n} s\right)=g(s), \quad s \in E, n \in \mathbb{Z},
$$

and $\tilde{g}(0)=0$. We have $\|\tilde{g}\|_{\infty}=\|g\|_{\infty}$. Conversely, if $h$ is any function satisfying $h(s)=h(2 s)$ a.e., then $h$ is uniquely (a.e.) determined by its restriction to $E$. This 1-1 mapping $g \rightarrow M_{\tilde{g}}$ from $L^{\infty}(E)$ onto $\{\widehat{D}, \widehat{T}\}^{\prime}$ is a *-isomorphism.

We will refer to a function $h$ satisfying $h(s)=h(2 s)$ a.e. as a 2-dilation periodic function. This gives a simple algorithm for computing a large class of wavelets from a given one, by simply modifying the phase (see also section 4.7):

Given $\psi$, let $\widehat{\psi}=\mathcal{F}(\psi)$, choose a real-valued function $h \in L^{\infty}(E)$

arbitrarily, let $g=\exp (i h)$, extend to a 2-dilation periodic

function $\tilde{g}$ as above, and compute $\psi_{\tilde{g}}=\mathcal{F}^{-1}(\tilde{g} \widehat{\psi})$.

In the description above, the set $E$ could clearly be replaced with $[-2 \pi,-\pi) \cup$ $[\pi, 2 \pi)$, or with any other "dyadic" set $[-2 a, a) \cup[a, 2 a)$ for some $a>0$.

\subsection{The Shannon Wavelet}

We now give an account of $s$-elementary and $M S F$-wavelets. The two most elementary dyadic orthonormal wavelets are the well-known Haar wavelet and Shannon's wavelet (also called the Littlewood-Paley wavelet). The Shannon set is the prototype of the class of wavelet sets.

Shannon's wavelet is the $L^{2}(\mathbb{R})$-function with Fourier transform $\widehat{\psi}_{S}=\frac{1}{\sqrt{2 \pi}} \chi_{E_{0}}$ where

$$
E_{0}=[-2 \pi,-\pi) \cup[\pi, 2 \pi) .
$$

The argument that $\widehat{\psi}_{S}$ is a wavelet is in a way even more transparent than for the Haar wavelet. And it has the advantage of generalizing nicely. For a simple argument, start from the fact that the exponents

$$
\left\{e^{i \ell s}: n \in \mathbb{Z}\right\}
$$

restricted to $[0,2 \pi]$ and normalized by $\frac{1}{\sqrt{2 \pi}}$ is an orthonormal basis for $L^{2}[0,2 \pi]$. Write $E_{0}=E_{-} \cup E_{+}$where $E_{-}=[-2 \pi,-\pi), E_{+}=[\pi, 2 \pi)$. Since $\left\{E_{-}+2 \pi, E_{+}\right\}$is a partition of $[0,2 \pi)$ and since the exponentials $e^{i \ell s}$ are invariant under translation by $2 \pi$, it follows that

$$
\left\{\left.\frac{e^{i \ell s}}{\sqrt{2 \pi}}\right|_{E_{0}}: n \in \mathbb{Z}\right\}
$$

is an orthonormal basis for $L^{2}\left(E_{0}\right)$. Since $\widehat{T}=M_{e^{-i s}}$, this set can be written

$$
\left\{\widehat{T}^{\ell} \widehat{\psi}_{s}: \ell \in \mathbb{Z}\right\} \text {. }
$$

Next, note that any "dyadic interval" of the form $J=[b, 2 b)$, for some $b>0$ has the property that $\left\{2^{n} J: n \in \mathbb{Z}\right\}$, is a partition of $(0, \infty)$. Similarly, any set of the form

$$
\mathcal{K}=[-2 a,-a) \cup[b, 2 b)
$$


for $a, b>0$, has the property that

$$
\left\{2^{n} \mathcal{K}: n \in \mathbb{Z}\right\}
$$

is a partition of $\mathbb{R} \backslash\{0\}$. It follows that the space $L^{2}(\mathcal{K})$, considered as a subspace of $L^{2}(\mathbb{R})$, is a complete wandering subspace for the dilation unitary $(D f)(s)=$ $\sqrt{2} f(2 s)$. For each $n \in \mathbb{Z}$,

$$
D^{n}\left(L^{2}(\mathcal{K})\right)=L^{2}\left(2^{-n} \mathcal{K}\right) .
$$

So $\bigoplus_{n} D^{n}\left(L^{2}(\mathcal{K})\right)$ is a direct sum decomposition of $L^{2}(\mathbb{R})$. In particular $E_{0}$ has this property. So

$$
D^{n}\left\{\left.\frac{e^{i \ell s}}{\sqrt{2 \pi}}\right|_{E_{0}}: \ell \in \mathbb{Z}\right\}=\left\{\left.\frac{e^{2^{n} i \ell s}}{\sqrt{2 \pi}}\right|_{2^{-n} E_{0}}: \ell \in \mathbb{Z}\right\}
$$

is an orthonormal basis for $L^{2}\left(2^{-n} E_{0}\right)$ for each $n$. It follows that

$$
\left\{D^{n} \widehat{T}^{\ell} \widehat{\psi}_{s}: n, \ell \in \mathbb{Z}\right\}
$$

is an orthonormal basis for $L^{2}(\mathbb{R})$. Hence $\left\{D^{n} T^{\ell} \psi_{s}: n, \ell \in \mathbb{Z}\right\}$ is an orthonormal basis for $L^{2}(\mathbb{R})$, as required.

For our work, in order to proceed with developing an operator-algebraic theory that had a chance of directly impacting concrete function-theoretic wavelet theory we needed a large supply of examples of wavelets which were elementary enough to work with. First, we found another "Shannon-type" wavelet in the literature. This was the Journe wavelet, which we found described on p. 136 in Daubechies book [8]. Its Fourier transform is $\widehat{\psi}_{J}=\frac{1}{\sqrt{2 \pi}} \chi_{E_{J}}$, where

$$
E_{J}=\left[-\frac{32 \pi}{7},-4 \pi\right) \cup\left[-\pi,-\frac{4 \pi}{7}\right) \cup\left[\frac{4 \pi}{7}, \pi\right) \cup\left[4 \pi, \frac{32 \pi}{7}\right) .
$$

Then, thinking the old adage "where there's smoke there's fire!", we painstakingly worked out many more examples. So far, these are the basic building blocks in the concrete part of our theory. By this we mean the part of our theory that has had some type of direct impact on function-theoretic wavelet theory.

\subsection{Definition of Wavelet Set}

We define a wavelet set to be a measurable subset $E$ of $\mathbb{R}$ for which $\frac{1}{\sqrt{2 \pi}} \chi_{E}$ is the

Fourier transform of a wavelet. The wavelet $\widehat{\psi}_{E}:=\frac{1}{\sqrt{2 \pi}} \chi_{E}$ is called s-elementary in $[5]$.

It turns out that this class of wavelets was also discovered and systematically explored completely independently, and in about the same time period, by Guido Weiss (Washington University), his colleague and former student E. Hernandez (U. Madrid), and his students X. Fang and X. Wang. Two of the papers of this group are [9] and [17], in which they are called MSF (minimally supported frequency) wavelets. In signal processing, the parameter $s$, which is the independent variable for $\widehat{\psi}$, is the frequency variable, and the variable $t$, which is the independent variable for $\psi$, is the time variable. It is not hard to show that no function with 
support a subset of a wavelet set $E$ of strictly smaller measure can be the Fourier transform of a wavelet. (Here, the support of a measurable function is defined to be the set of points at which it does not vanish.) In other words, an MSF wavelet has minimal possible support in the frequency domain. However, the problem of whether the support set of any wavelet necessarily contains a wavelet set remains open. It was raised by this author (Larson) in a talk about ten years ago, and has been open for several years. We include it as Problem 3 in the final section of this article. A natural subproblem, which was posed in the same talk, asks whether a wavelet with minimal possible support in the frequency domain is in fact an MSF wavelet; or equivalently, is its support a wavelet set?

4.4.1. The Spectral Set Condition. ¿From the argument above describing why Shannon's wavelet is, indeed, a wavelet, it is clear that sufficient conditions for $E$ to be a wavelet set are

(i) the normalized exponential $\frac{1}{\sqrt{2 \pi}} e^{i \ell s}, \ell \in \mathbb{Z}$, when restricted to $E$ should constitute an orthonormal basis for $L^{2}(E)$ (in other words $E$ is a spectral set for the integer lattice $\mathbb{Z}$ ),

and

(ii) The family $\left\{2^{n} E: \quad n \in \mathbb{Z}\right\}$ of dilates of $E$ by integral powers of 2 should constitute a measurable partition (i.e. a partition modulo null sets) of $\mathbb{R}$.

These conditions are also necessary. In fact if a set $E$ satisfies (i), then for it to be a wavelet set it is obvious that (ii) must be satisfied. To show that (i) must be satisfied by a wavelet set $E$, consider the vectors

$$
\widehat{D}^{n} \widehat{\psi}_{E}=\frac{1}{\sqrt{2 \pi}} \chi_{2^{-n} E}, \quad n \in \mathbb{Z}
$$

Since $\widehat{\psi}_{E}$ is a wavelet these must be orthogonal, and so the sets $\left\{2^{n} E: n \in \mathbb{Z}\right\}$ must be disjoint modulo null sets. It follows that $\left\{\left.\frac{1}{\sqrt{2 \pi}} e^{i \ell s}\right|_{E}: \quad \ell \in \mathbb{Z}\right\}$ is not only an orthonormal set of vectors in $L^{2}(E)$, it must also span $L^{2}(E)$.

It is known from the theory of spectral sets (as an elementary special case) that a measurable set $E$ satisfies (i) if and only if it is a generator of a measurable partition of $\mathbb{R}$ under translation by $2 \pi$ (i.e. iff $\{E+2 \pi n: n \in \mathbb{Z}\}$ is a measurable partition of $\mathbb{R}$ ). This result generalizes to spectral sets for the integral lattice in $\mathbb{R}^{n}$. For this elementary special case a direct proof is not hard.

\subsection{Translation and Dilation Congruence}

We say that measurable sets $E, F$ are translation congruent modulo $2 \pi$ if there is a measurable bijection $\phi: E \rightarrow F$ such that $\phi(s)-s$ is an integral multiple of $2 \pi$ for each $s \in E$; or equivalently, if there is a measurable partition $\left\{E_{n}: n \in \mathbb{Z}\right\}$ of E such that

$$
\left\{E_{n}+2 n \pi: n \in \mathbb{Z}\right\}
$$


is a measurable partition of $F$. Analogously, define measurable sets $G$ and $H$ to be dilation congruent modulo 2 if there is a measurable bijection $\tau: G \rightarrow H$ such that for each $s \in G$ there is an integer $n$, depending on $s$, such that $\tau(s)=2^{n} s$; or equivalently, if there is a measurable partition $\left\{G_{n}\right\}_{-\infty}^{\infty}$ of $G$ such that

$$
\left\{2^{n} G\right\}_{-\infty}^{\infty}
$$

is a measurable partition of $H$. (Translation and dilation congruency modulo other positive numbers of course make sense as well.)

The following lemma is useful.

Lemma 4.2. Let $f \in L^{2}(\mathbb{R})$, and let $E=\operatorname{supp}(f)$. Then $f$ has the property that

$$
\left\{e^{i n s} f: n \in \mathbb{Z}\right\}
$$

is an orthonormal basis for $L^{2}(E)$ if and only if

(i) $E$ is congruent to $[0,2 \pi)$ modulo $2 \pi$, and

(ii) $|f(s)|=\frac{1}{\sqrt{2 \pi}}$ a.e. on $E$.

If $E$ is a measurable set which is $2 \pi$-translation congruent to $[0,2 \pi)$, then since

$$
\left\{\left.\frac{e^{i \ell s}}{\sqrt{2 \pi}}\right|_{[0,2 \pi)}: \ell \in \mathbb{Z}\right\}
$$

is an orthonormal basis for $L^{2}[0,2 \pi]$ and the exponentials $e^{i \ell s}$ are $2 \pi$-invariant, as in the case of Shannon's wavelet it follows that

$$
\left\{\left.\frac{e^{i \ell s}}{\sqrt{2 \pi}}\right|_{E}: \ell \in \mathbb{Z}\right\}
$$

is an orthonormal basis for $L^{2}(E)$. Also, if $E$ is $2 \pi$-translation congruent to $[0,2 \pi)$, then since

$$
\{[0,2 \pi)+2 \pi n: \quad n \in \mathbb{Z}\}
$$

is a measurable partition of $\mathbb{R}$, so is

$$
\{E+2 \pi n: n \in \mathbb{Z}\} .
$$

These arguments can be reversed.

We say that a measurable subset $G \subset \mathbb{R}$ is a 2-dilation generator of a partition of $\mathbb{R}$ if the sets

$$
2^{n} G:=\left\{2^{n} s: s \in G\right\}, \quad n \in \mathbb{Z}
$$

are disjoint and $\mathbb{R} \backslash \cup_{n} 2^{n} G$ is a null set. Also, we say that $E \subset \mathbb{R}$ is a $2 \pi$-translation generator of a partition of $\mathbb{R}$ if the sets

$$
E+2 n \pi:=\{s+2 n \pi: s \in E\}, \quad n \in \mathbb{Z},
$$

are disjoint and $\mathbb{R} \backslash \cup_{n}(E+2 n \pi)$ is a null set. 
Lemma 4.3. A measurable set $E \subseteq \mathbb{R}$ is a $2 \pi$-translation generator of a partition of $\mathbb{R}$ if and only if, modulo a null set, $E$ is translation congruent to $[0,2 \pi)$ modulo $2 \pi$. Also, a measurable set $G \subseteq \mathbb{R}$ is a 2-dilation generator of a partition of $\mathbb{R}$ if and only if, modulo a null set, $G$ is a dilation congruent modulo 2 to the set $[-2 \pi,-\pi) \cup[\pi, 2 \pi)$.

\subsection{A Criterion}

The following is a useful criterion for wavelet sets. It was published independently by Dai-Larson in [5] and by Fang-Wang (who were students of Guido Weiss) in [9] at about the same time (in December, 1994). In fact, it is amusing that the two papers had been submitted within two days of each other; only much later did we even learn of each other's work on wavelets and of this incredible timing.

Proposition 4.4. Let $E \subseteq \mathbb{R}$ be a measurable set. Then $E$ is a wavelet set if and only if $E$ is both a 2-dilation generator of a partition (modulo null sets) of $\mathbb{R}$ and a $2 \pi$-translation generator of a partition (modulo null sets) of $\mathbb{R}$. Equivalently, $E$ is a wavelet set if and only if $E$ is both translation congruent to $[0,2 \pi)$ modulo $2 \pi$ and dilation congruent to $[-2 \pi,-\pi) \cup[\pi, 2 \pi)$ modulo 2 .

Note that a set is $2 \pi$-translation congruent to $[0,2 \pi)$ iff it is $2 \pi$-translation congruent to $[-2 \pi, \pi) \cup[\pi, 2 \pi)$. So the last sentence of Proposition 4.4 can be stated: A measurable set $E$ is a wavelet set if and only if it is both $2 \pi$-translation and 2-dilation congruent to the Littlewood-Paley set $[-2 \pi,-\pi) \cup[\pi, 2 \pi)$.

\subsection{Phases}

If $E$ is a wavelet set, and if $f(s)$ is any function with support $E$ which has constant modulus $\frac{1}{\sqrt{2 \pi}}$ on $E$, then $\mathcal{F}^{-1}(f)$ is a wavelet. Indeed, by Lemma $4.2\left\{\widehat{T}^{\ell} f: \in \mathbb{Z}\right\}$ is an orthonormal basis for $L^{2}(E)$, and since the sets $2^{n} E$ partition $\mathbb{R}$, so $L^{2}(E)$ is a complete wandering subspace for $\widehat{D}$, it follows that $\left\{\widehat{D}^{n} \widehat{T}^{\ell} f: n, \ell \in \mathbb{Z}\right\}$ must be an orthonormal basis for $L^{2}(\mathbb{R})$, as required. In $[9,17]$ the term MSF-wavelet includes this type of wavelet. So MSF-wavelets can have arbitrary phase and $s$ elementary wavelets have phase 0 . If $\psi$ is a wavelet we say [5] that a real-valued function $\rho(s)$ is attainable as a phase of $\psi$ if the function $e^{i \rho(s)}|\psi(s)|$ is also the Fourier transform of a wavelet. So every phase is attainable in this sense for an MSF or $s$-elementary wavelet. Attainable phases of wavelets have been studied in [5] and [26], in particular.

\subsection{Some Examples of One-Dimensional Wavelet Sets}

It is usually easy to determine, using the dilation-translation criteria, in Proposition 4.4 whether a given finite union of intervals is a wavelet set. In fact, to verify that a given "candidate" set $E$ is a wavelet set, it is clear from the above discussion and criteria that it suffices to do two things.

(1) Show, by appropriate partitioning, that $E$ is 2-dilation-congruent to a set of the form $[-2 a,-a) \cup[b, 2 b)$ for some $a, b>0$.

and 
(2) Show, by appropriate partitioning, that $E$ is $2 \pi$-translation-congruent to a set of the form $[c, c+2 \pi)$ for some real number $c$.

On the other hand, wavelet sets suitable for testing hypotheses can be quite difficult to construct. There are very few "recipes" for wavelet sets, as it were. Many families of such sets have been constructed for reasons including perspective, experimentation, testing hypotheses, etc., including perhaps the pure enjoyment of doing the computations - which are somewhat "puzzle-like" in nature. In working with the theory it is nice (and in fact we find it necessary) to have a large supply of wavelets on hand that permit relatively simple analysis.

For this reason we take the opportunity here to present for the reader a collection of such sets, mainly taken from [5], leaving most of the work in verifying that they are indeed wavelet sets to the reader.

We refer the reader to [6] for a proof of the existence of wavelet sets in $\mathbb{R}^{(n)}$, and a proof that there are sufficiently many to generate the Borel structure of $\mathbb{R}^{(n)}$. These results are true for arbitrary expansive dilation factors. Some concrete examples in the plane were subsequently obtained by Soardi and Weiland, and others were obtained by Gu and by Speegle in their thesis work at A\&M. Two had also been obtained by Dai for inclusion in the revised concluding remarks section of our Memoir [5].

In these examples we will usually write intervals as half-open intervals $[\cdot$, ) because it is easier to verify the translation and dilation congruency relations (1) and (2) above when wavelet sets are written thus, even though in actuality the relations need only hold modulo null sets.

(i) As mentioned above, an example due to Journe of a wavelet which admits no multiresolution analysis is the $s$-elementary wavelet with wavelet set

$$
\left[-\frac{32 \pi}{7},-4 \pi\right) \cup\left[-\pi, \frac{4 \pi}{7}\right) \cup\left[\frac{4 \pi}{7}, \pi\right) \cup\left[4 \pi, \frac{32 \pi}{7}\right) .
$$

To see that this satisfies the criteria, label these intervals, in order, as $J_{1}, J_{2}, J_{3}, J_{4}$ and write $J=\cup J_{i}$. Then

$$
J_{1} \cup 4 J_{2} \cup 4 J_{3} \cup J_{4}=\left[-\frac{32 \pi}{7},-\frac{16 \pi}{7}\right) \cup\left[\frac{16 \pi}{7}, \frac{32 \pi}{7}\right) .
$$

This has the form $[-2 a, a) \cup[b, 2 b)$ so is a 2-dilation generator of a partition of $\mathbb{R} \backslash\{0\}$. Then also observe that

$$
\left\{J_{1}+6 \pi, J_{2}+2 \pi, J_{3}, J_{4}-4 \pi\right\}
$$

is a partition of $[0,2 \pi)$.

(ii) The Shannon (or Littlewood-Paley) set can be generalized. For any $-\pi<$ $\alpha<\pi$, the set

$$
E_{\alpha}=[-2 \pi+2 \alpha,-\pi+\alpha) \cup[\pi+\alpha, 2 \pi+2 \alpha)
$$

is a wavelet set. Indeed, it is clearly a 2-dilation generator of a partition of $\mathbb{R} \backslash\{0\}$, and to see that it satisfies the translation congruency criterion for $-\pi<\alpha \leq 0$ 
(the case $0<\alpha<\pi$ is analogous) just observe that

$$
\{[-2 \pi+2 \alpha, 2 \pi)+4 \pi,[-2 \pi,-\pi+\alpha)+2 \pi,[\pi+\alpha, 2 \pi+2 \alpha)\}
$$

is a partition of $[0,2 \pi)$. It is clear that $\psi_{E_{\alpha}}$ is then a continuous (in $L^{2}(\mathbb{R})$-norm) path of $s$-elementary wavelets. Note that

$$
\lim _{\alpha \rightarrow \pi} \widehat{\psi}_{E_{\alpha}}=\frac{1}{\sqrt{2 \pi}} \chi_{[2 \pi, 4 \pi)} .
$$

This is not the Fourier transform of a wavelet because the set $[2 \pi, 4 \pi)$ is not a 2-dilation generator of a partition of $\mathbb{R} \backslash\{0\}$. So

$$
\lim _{\alpha \rightarrow \pi} \psi_{E_{\alpha}}
$$

is not an orthogonal wavelet. (It is what is known as a Hardy wavelet because it generates an orthonormal basis for $H^{2}(\mathbb{R})$ under dilation and translation.) This example demonstrates that $\mathcal{W}(D, T)$ is not closed in $L^{2}(\mathbb{R})$. set

(iii) Journe's example above can be extended to a path. For $-\frac{\pi}{7} \leq \beta \leq \frac{\pi}{7}$ the

$J_{\beta}=\left[-\frac{32 \pi}{7},-4 \pi+4 \beta\right) \cup\left[-\pi+\beta,-\frac{4 \pi}{7}\right) \cup\left[\frac{4 \pi}{7}, \pi+\beta\right) \cup\left[4 \pi+4 \beta, 4 \pi+\frac{4 \pi}{7}\right)$

is a wavelet set. The same argument in (i) establishes dilation congruency. For translation, the argument in (i) shows congruency to $[4 \beta, 2 \pi+4 \beta)$ which is in turn congruent to $[0,2 \pi)$ as required. Observe that here, as opposed to in (ii) above, the limit of $\psi_{J_{\beta}}$ as $\beta$ approaches the boundary point $\frac{\pi}{7}$ is a wavelet. Its wavelet set is a union of 3 disjoint intervals.

(iv) Let $A \subseteq\left[\pi, \frac{3 \pi}{2}\right)$ be an arbitrary measurable subset. Then there is a wavelet set $W$, such that $W \cap\left[\pi, \frac{3 \pi}{2}\right)=A$. For the construction, let

$$
\begin{aligned}
B & =[2 \pi, 3 \pi) \backslash 2 A, \\
C & =\left[-\pi,-\frac{\pi}{2}\right) \backslash(A-2 \pi) \\
\text { and } \quad D & =2 A-4 \pi .
\end{aligned}
$$

Let

$$
W=\left[\frac{3 \pi}{2}, 2 \pi\right) \cup A \cup B \cup C \cup D .
$$

We have $W \cap\left[\pi, \frac{3 \pi}{2}\right)=A$. Observe that the sets $\left[\frac{3 \pi}{2}, 2 \pi\right), A, B, C, D$, are disjoint. Also observe that the sets

$$
\left[\frac{3 \pi}{2}, 2 \pi\right), A, \frac{1}{2} B, 2 C, D
$$

are disjoint and have union $[-2 \pi,-\pi) \cup[\pi, 2 \pi)$. In addition, observe that the sets

$$
\left[\frac{3 \pi}{2}, 2 \pi\right), A, B-2 \pi, C+2 \pi, D+2 \pi,
$$

are disjoint and have union $[0,2 \pi)$. Hence $W$ is a wavelet set. 
(v) Wavelet sets for arbitrary (not necessarily integral) dilation factors other then 2 exist. For instance, if $d \geq 2$ is arbitrary, let

$$
\begin{aligned}
& A=\left[-\frac{2 d \pi}{d+1},-\frac{2 \pi}{d+1}\right), \\
& B=\left[\frac{2 \pi}{d^{2}-1}, \frac{2 \pi}{d+1}\right), \\
& C=\left[\frac{2 d \pi}{d+1}, \frac{2 d^{2} \pi}{d^{2}-1}\right)
\end{aligned}
$$

and let $G=A \cup B \cup C$. Then $G$ is $d$-wavelet set. To see this, note that $\{A+2 \pi, B, C\}$ is a partition of an interval of length $2 \pi$. So $G$ is $2 \pi$-translation-congruent to $[0,2 \pi)$. Also, $\left\{A, B, d^{-1} C\right\}$ is a partition of the set $[-d \alpha,-\alpha) \cup[\beta, d \beta)$ for $\alpha=\frac{2 \pi}{d^{2}-1}$, and $\beta=\frac{2 \pi}{d^{2}-1}$, so from this form it follows that $\left\{d^{n} G: n \in \mathbb{Z}\right\}$ is a partition of $\mathbb{R} \backslash\{0\}$. Hence if $\psi:=\mathcal{F}^{-1}\left(\frac{1}{\sqrt{2 \pi}} \chi_{G}\right)$, it follows that $\left\{d^{\frac{n}{2}} \psi\left(d^{n} t-\ell\right): n, \ell \in \mathbb{Z}\right\}$ is orthonormal basis for $L^{2}(\mathbb{R})$, as required.

\section{Operator-Theoretic Interpolation for Wavelet Sets}

Operator-theoretic interpolation takes a particularly natural form for the special case of s-elementary (or MSF) wavelets that facilitates hands-on computational techniques in investigating its properties. Let $E, F$ be a pair of wavelet sets. Then for (a.e.) $x \in E$ there is a unique $y \in F$ such that $x-y \in 2 \pi \mathbb{Z}$. This is the translation congruence property of wavelet sets. Also, for (a.e.) $x \in E$ there is a unique $z \in F$ such that $\frac{x}{z}$ is an integral power of 2 . This is the dilation congruence property of wavelet sets. (See section 2.5.6.)

There is a natural closed-form algorithm for the interpolation unitary $V_{\psi_{E}}^{\psi_{F}}$ which maps the wavelet basis for $\widehat{\psi}_{E}$ to the wavelet basis for $\widehat{\psi}_{F}$. Indeed, using both the translation and dilation congruence properties of $\{E, F\}$, one can explicitly compute a (unique) measure-preserving transformation $\sigma:=\sigma_{E}^{F}$ mapping $\mathbb{R}$ onto $\mathbb{R}$ which has the property that $V_{\psi_{E}}^{\psi_{F}}$ is identical with the composition operator defined by:

$$
f \mapsto f \circ \sigma^{-1}
$$

for all $f \in L^{2}(\mathbb{R})$. With this formulation, compositions of the maps $\sigma$ between different pairs of wavelet sets are not difficult to compute, and thus products of the corresponding interpolation unitaries can be computed in terms of them.

\subsection{The Interpolation Map $\sigma$}

Let $E$ and $F$ be arbitrary wavelet sets. Let $\sigma: E \rightarrow F$ be the 1-1, onto map implementing the $2 \pi$-translation congruence. Since $E$ and $F$ both generated partitions of $\mathbb{R} \backslash\{0\}$ under dilation by powers of 2 , we may extend $\sigma$ to a 1-1 map of $\mathbb{R}$ onto $\mathbb{R}$ by defining $\sigma(0)=0$, and

$$
\sigma(s)=2^{n} \sigma\left(2^{-n} s\right) \quad \text { for } \quad s \in 2^{n} E, \quad n \in \mathbb{Z} .
$$


We adopt the notation $\sigma_{E}^{F}$ for this, and call it the interpolation map for the ordered pair $(E, F)$.

Lemma 5.1. In the above notation, $\sigma_{E}^{F}$ is a measure-preserving transformation from $\mathbb{R}$ onto $\mathbb{R}$.

Proof. Let $\sigma:=\sigma_{E}^{F}$. Let $\Omega \subseteq \mathbb{R}$ be a measurable set. Let $\Omega_{n}=\Omega \cap 2^{n} E, n \ni \mathbb{Z}$, and let $E_{n}=2^{-n} \Omega_{n} \subseteq E$. Then $\left\{\Omega_{n}\right\}$ is a partition of $\Omega$, and we have $m\left(\sigma\left(E_{n}\right)\right)=$ $m\left(E_{n}\right)$ because the restriction of $\sigma$ to $E$ is measure-preserving. So

$$
\begin{aligned}
m(\sigma(\Omega)) & =\sum_{n} m\left(\sigma\left(\Omega_{n}\right)\right)=\sum_{n} m\left(2^{n} \sigma\left(E_{n}\right)\right) \\
& =\sum_{n} 2^{n} m\left(\sigma\left(E_{n}\right)\right)=\sum_{n} 2^{n} m\left(E_{n}\right) \\
& =\sum_{n} m\left(2^{n} E_{n}\right)=\sum_{n} m\left(\Omega_{n}\right)=m(\Omega) .
\end{aligned}
$$

A function $f: \mathbb{R} \rightarrow \mathbb{R}$ is called 2-homogeneous if $f(2 s)=2 f(s)$ for all $s \in \mathbb{R}$. Equivalently, $f$ is 2-homogeneous iff $f\left(2^{n} s\right)=2^{n} f(s), s \in \mathbb{R}, n \in \mathbb{Z}$. Such a function is completely determined by its values on any subset of $\mathbb{R}$ which generates a partition of $\mathbb{R} \backslash\{0\}$ by 2-dilation. So $\sigma_{E}^{F}$ is the (unique) 2-homogeneous extension of the $2 \pi$-transition congruence $E \rightarrow F$. The set of all 2-homogeneous measurepreserving transformations of $\mathbb{R}$ clearly forms a group under composition. Also, the composition of a 2-dilation-periodic function $f$ with a 2-homogeneous function $g$ is (in either order) 2-dilation periodic. We have $f(g(2 s))=f(2 g(s))=f(g(s))$ and $g(f(2 s))=g(f(s))$. These facts will be useful.

\subsubsection{An Algorithm For The Interpolation Unitary. Now let}

$$
U_{E}^{F}:=U_{\sigma_{E}^{F}}
$$

where if $\sigma$ is any measure-preserving transformation of $\mathbb{R}$ then $U_{\sigma}$ denotes the composition operator defined by $U_{\sigma} f=f \circ \sigma^{-1}, f \in L^{2}(\mathbb{R})$. Clearly $\left(\sigma_{E}^{F}\right)^{-1}=\sigma_{F}^{E}$ and $\left(U_{E}^{F}\right)^{*}=U_{F}^{E}$. We have $U_{E}^{F} \widehat{\psi}_{E}=\widehat{\psi}_{F}$ since $\sigma_{E}^{F}(E)=F$. That is,

$$
U_{E}^{F} \widehat{\psi}_{E}=\widehat{\psi}_{E} \circ \sigma_{F}^{E}=\frac{1}{\sqrt{2 \pi}} \chi_{E} \circ \sigma_{F}^{E}=\frac{1}{\sqrt{2 \pi}} \chi_{F}=\widehat{\psi}_{F} .
$$

Proposition 5.2. Let $E$ and $F$ be arbitrary wavelet sets. Then $U_{E}^{F} \in \mathcal{C}_{\widehat{\psi}_{E}}(\widehat{D}, \widehat{T})$. Hence $\mathcal{F}^{-1} U_{E}^{F} \mathcal{F}$ is the interpolation unitary for the ordered pair $\left(\psi_{E}, \psi_{F}\right)$.

Proof. Write $\sigma=\sigma_{E}^{F}$ and $U_{\sigma}=U_{E}^{F}$. We have $U_{\sigma} \widehat{\psi}_{E}=\widehat{\psi}_{F}$ since $\sigma(E)=F$. We must show

$$
U_{\sigma} \widehat{D}^{n} \widehat{T}^{l} \widehat{\psi}_{E}=\widehat{D}^{n} \widehat{T}^{l} U_{\sigma} \widehat{\psi}_{E}, \quad n, l \in \mathbb{Z}
$$


We have

$$
\begin{aligned}
\left(U_{\sigma} \widehat{D}^{n} \widehat{T}^{l} \widehat{\psi}_{E}\right)(s) & =\left(U_{\sigma} \widehat{D}^{n} e^{-i l s} \widehat{\psi}_{E}\right)(s) \\
& =U_{\sigma} 2^{-\frac{n}{2}} e^{-i l 2^{-n} s} \widehat{\psi}_{E}\left(2^{-n} s\right) \\
& =2^{-\frac{n}{2}} e^{-i l 2^{-n} \sigma^{-1}(s)} \widehat{\psi}_{E}\left(2^{-n} \sigma^{-1}(s)\right) \\
& =2^{-\frac{n}{2}} e^{-i l \sigma^{-1}\left(2^{-n} s\right)} \widehat{\psi}_{E}\left(\sigma^{-1}\left(2^{-n} s\right)\right) \\
& =2^{-\frac{n}{2}} e^{-i l \sigma^{-1}\left(2^{-n} s\right)} \widehat{\psi}\left(2^{-n} s\right) .
\end{aligned}
$$

This last term is nonzero iff $2^{-n} s \in F$, in which case $\sigma^{-1}\left(2^{-n} s\right)=\sigma_{F}^{E}\left(2^{-n} s\right)$ $=2^{-n} s+2 \pi k$ for some $k \in \mathbb{Z}$ since $\sigma_{F}^{E}$ is a $2 \pi$-translation-congruence on $F$. It follows that $e^{-i l \sigma^{-2}\left(2^{-n} s\right)}=e^{-i l 2^{-n} s}$. Hence we have

$$
\begin{aligned}
\left(U_{\sigma} \widehat{D}^{n} \widehat{T}^{l} \widehat{\psi}_{E}\right)(s) & =2^{-\frac{n}{2}} e^{-i l s^{-2 n} s} \widehat{\psi}_{F}\left(2^{-n} s\right) \\
& =\left(\widehat{D}^{n} \widehat{T}^{l} \widehat{\psi}_{F}\right)(s) \\
& =\left(\widehat{D}^{n} \widehat{T}^{l} U_{\sigma} \widehat{\psi}_{E}\right)(s) .
\end{aligned}
$$

We have shown $U_{E}^{F} \in \mathcal{C}_{\widehat{\psi}_{E}}(\widehat{D}, \widehat{T})$. Since $U_{E}^{F} \widehat{\psi}_{E}=\widehat{\psi}_{F}$, the uniqueness part of Proposition 3.1 shows that $\mathcal{F}^{-1} U_{E}^{F} \mathcal{F}$ must be the interpolation unitary for $\left(\psi_{E}, \psi_{F}\right)$.

\subsection{The Interpolation Unitary Normalizes The Commutant}

Proposition 5.3. Let $E$ and $F$ be arbitrary wavelet sets. Then the interpolation unitary for the ordered pair $\left(\psi_{E}, \psi_{F}\right)$ normalizes $\{D, T\}^{\prime}$.

Proof. By Proposition 5.2 we may work with $U_{E}^{F}$ in the Fourier transform domain. By Theorem 6 , the generic element of $\{\widehat{D}, \widehat{T}\}^{\prime}$ has the form $M_{h}$ for some 2-dilationperiodic function $h \in L^{\infty}(\mathbb{R})$. Write $\sigma=\sigma_{E}^{F}$ and $U_{\sigma}=U_{E}^{F}$. Then

$$
U_{\sigma}^{-1} M_{h} U_{\sigma}=M_{h \circ \sigma^{-1}}
$$

So since the composition of a 2-dilation-periodic function with a 2-homogeneous function is 2-dilation-periodic, the proof is complete.

5.2.1. $\mathcal{C}_{\psi}(D, T)$ is Nonabelian. It can also be shown ([5, Theorem 5.2 (iii) $\left.]\right)$ that if $E, F$ are wavelet sets with $E \neq F$ then $U_{E}^{F}$ is not contained in the double commutant $\{\widehat{D}, \widehat{T}\}^{\prime \prime}$. So since $U_{E}^{F}$ and $\{\widehat{D}, \widehat{T}\}^{\prime}$ are both contained in the local commutant of $\mathcal{U}_{\widehat{D}, \widehat{T}}$ at $\widehat{\psi}_{E}$, this proves that $\mathcal{C}_{\widehat{\psi}_{E}}(\widehat{D}, \widehat{T})$ is nonabelian. In fact (see $\left[5\right.$, Proposition 1.8]) this can be used to show that $\mathcal{C}_{\psi}(D, T)$ is nonabelian for every wavelet $\psi$. We suspected this, but we could not prove it until we discovered the "right" way of doing the needed computation using $s$-elementary wavelets.

The above shows that a pair $(E, F)$ of wavelets sets (or, rather, their corresponding $s$-elementary wavelets) admits operator-theoretic interpolation if and only if Group $\left\{U_{E}^{F}\right\}$ is contained in the local commutant $\mathcal{C}_{\widehat{\psi}_{E}}(\widehat{D}, \widehat{T})$, since the requirement that $U_{E}^{F}$ normalizes $\{\widehat{D}, \widehat{T}\}^{\prime}$ is automatically satisfied. It is easy to see that this is equivalent to the condition that for each $n \in \mathbb{Z}, \sigma^{n}$ is a $2 \pi$-congruence 
of $E$ in the sense that $\left(\sigma^{n}(s)-s\right) / 2 \pi \in \mathbb{Z}$ for all $s \in E$, which in turn implies that $\sigma^{n}(E)$ is a wavelet set for all $n$. Here $\sigma=\sigma_{E}^{F}$. This property hold trivially if $\sigma$ is involutive (i.e. $\sigma^{2}=$ identity).

5.2.2. The Coefficient Criterion. In cases where "torsion" is present, so $\left(\sigma_{E}^{F}\right)^{k}$ is the identity map for some finite integer $k$, the von Neumann algebra generated by $\{\widehat{D}, \widehat{T}\}^{\prime}$ and $U:=U_{E}^{F}$ has the simple form

$$
\left\{\sum_{n=0}^{k} M_{h_{n}} U^{n}: h_{n} \in L^{\infty}(\mathbb{R}) \text { with } h_{n}(2 s)=h_{n}(s), \quad s \in \mathbb{R}\right\},
$$

and so each member of this "interpolated" family of wavelets has the form

$$
\frac{1}{\sqrt{2 \pi}} \sum_{n=0}^{k} h_{n}(s) \chi_{\sigma^{n}(E)}
$$

for 2-dilation periodic "coefficient" functions $\left\{h_{n}(s)\right\}$ which satisfy the necessary and sufficient condition that the operator

$$
\sum_{n=0}^{k} M_{h_{n}} U^{n}
$$

is unitary.

A standard computation shows that the map $\theta$ sending $\sum_{0}^{k} M_{h_{n}} U^{n}$ to the $k \times k$ function matrix $\left(h_{i j}\right)$ given by

$$
h_{i j}=h_{\alpha(i, j)} \circ \sigma^{-i+1}
$$

where $\alpha(i, j)=(i+1)$ modulo $k$, is a $*$-isomorphism. This matricial algebra is the cross-product of $\{D, T\}^{\prime}$ by the *-automorphism $\operatorname{ad}\left(U_{E}^{F}\right)$ corresponding to conjugation with $U_{E}^{F}$. For instance, if $k=3$ then $\theta$ maps

$$
M_{h_{1}}+M_{h_{2}} U_{E}^{F}+M_{h_{3}}\left(U_{E}^{F}\right)^{2}
$$

to

$$
\left(\begin{array}{ccc}
h_{1} & h_{2} & h_{3} \\
h_{3} \circ \sigma^{-1} & h_{1} \circ \sigma^{-1} & h_{2} \circ \sigma^{-1} \\
h_{2} \circ \sigma^{-2} & h_{3} \circ \sigma^{-2} & h_{1} \circ \sigma^{-2}
\end{array}\right) .
$$

This shows that $\sum_{0}^{k} M_{h_{n}} U^{n}$ is a unitary operator iff the scalar matrix $\left(h_{i j}\right)(s)$ is unitary for almost all $s \in \mathbb{R}$. Unitarity of this matrix-valued function is called the Coefficient Criterion in [5], and the functions $h_{i}$ are called the interpolation coefficients. This leads to formulas for families of wavelets which are new to wavelet theory. 


\subsection{Interpolation Pairs of Wavelet Sets}

For many interesting cases of note, the interpolation map $\sigma_{E}^{F}$ will in fact be an involution of $\mathbb{R}$ (i.e. $\sigma \circ \sigma=i d$, where $\sigma:=\sigma_{E}^{F}$, and where $i d$ denotes the identity map). So torsion will be present, as in the above section, and it will be present in an essentially simple form. The corresponding interpolation unitary will be a symmetry in this case (i.e. a selfadjoint unitary operator with square $I$ ).

It is curious to note that verifying a simple operator equation $U^{2}=I$ directly by matricial computation can be extremely difficult. It is much more computationally feasible to verify an equation such as this by pointwise (a.e.) verifying explicitly the relation $\sigma \circ \sigma=i d$ for the interpolation map. In [5] we gave a number of examples of interpolation pairs of wavelet sets. We give below a collection of examples that has not been previously published: Every pair sets from the Journe family is an interpolation pair.

\subsection{Journe Family Interpolation Pairs}

Consider the parameterized path of generalized Journe wavelet sets given in Section 4.8 Item (iii). We have

$J_{\beta}=\left[-\frac{32 \pi}{7},-4 \pi-4 \beta\right) \cup\left[-\pi+\beta,-\frac{4 \pi}{7}\right) \cup\left[\frac{4 \pi}{7}, \pi+\beta\right) \cup\left[4 \pi+4 \beta, 4 \pi+\frac{4 \pi}{7}\right)$

where the set of parameters $\beta$ ranges $-\frac{\pi}{7} \leq \beta \leq \frac{\pi}{7}$.

Proposition 5.4. Every pair $\left(J_{\beta_{1}}, J_{\beta_{2}}\right)$ is an interpolation pair.

Proof. Let $\beta_{1}, \beta_{2} \in\left[-\frac{\pi}{7}, \frac{\pi}{7}\right)$ with $\beta_{1}<\beta_{2}$. Write $\sigma=\sigma_{J_{\beta_{2}}}^{J_{\beta_{1}}}$. We need to show that

$$
\sigma^{2}(x)=x
$$

for all $x \in \mathbb{R}$. Since $\sigma$ is 2-homogeneous, it suffices to verify $\left(^{*}\right)$ only for $x \in J_{\beta_{1}}$. For $x \in J_{\beta_{1}} \cap J_{\beta_{2}}$ we have $\sigma(x)=x$, hence $\sigma^{2}(x)=x$. So we only need to check $(*)$ for $x \in\left(J_{\beta_{1}} \backslash J_{\beta_{2}}\right)$. We have

$$
J_{\beta_{1}} \backslash J_{\beta_{2}}=\left[-\pi+\beta_{1},-\pi+\beta_{2}\right) \cup\left[4 \pi+4 \beta_{1}, 4 \pi+4 \beta_{2}\right) .
$$

It is useful to also write

$$
J_{\beta_{2}} \backslash J_{\beta_{1}}=\left[-4 \pi+4 \beta_{1},-4 \pi+4 \beta_{2}\right) \cup\left[\pi+\beta_{1}, \pi+\beta_{2}\right) .
$$

On $\left[-\pi+\beta_{1},-\pi+\beta_{2}\right)$ we have $\sigma(x)=x+2 \pi$, which lies in $\left[\pi+\beta_{1}, \pi+\beta_{2}\right)$. If we multiply this by 4 , we obtain $4 \sigma(x) \in\left[4 \pi+4 \beta_{1}, 4 \pi+4 \beta_{2}\right) \subset J_{\beta_{1}}$. And on $\left[4 \pi+4 \beta_{1}, 4 \pi+4 \beta_{2}\right)$ we clearly have $\sigma(x)=x-8 \pi$, which lies in $\left[-4 \pi+4 \beta_{1},-4 \pi+\right.$ $\left.4 \beta_{2}\right)$.

So for $x \in\left[-\pi+\beta_{1},-\pi+\beta_{2}\right)$ we have

$$
\sigma^{2}(x)=\sigma(\sigma(x))=\frac{1}{4} \sigma(4 \sigma(x))=\frac{1}{4}[4 \sigma(x)-8 \pi]=\sigma(x)-2 \pi=x+2 \pi-2 \pi=x .
$$


On $\left[4 \pi+4 \beta_{1}, 4 \pi+4 \beta_{2}\right)$ we have $\sigma(x)=x-8 \pi$, which lies in $\left[-4 \pi+4 \beta_{1},-4 \pi+\right.$ $\left.4 \beta_{2}\right)$. So $\frac{1}{4} \sigma(x) \in\left[-\pi+\beta_{1},-\pi+\beta_{2}\right)$. Hence

$$
\sigma\left(\frac{1}{4} \sigma(x)\right)=\frac{1}{4} \sigma(x)+2 \pi
$$

and thus

$$
\sigma^{2}(x)=4 \sigma\left(\frac{1}{4} \sigma(x)\right)=4\left[\frac{1}{4} \sigma(x)+2 \pi\right]=\sigma(x)+8 \pi=x-8 \pi+8 \pi=x
$$

as required.

We have shown that for all $x \in J_{\beta_{1}}$ we have $\sigma^{2}(x)=x$. This proves that $\left(J_{\beta_{1}}, J_{\beta_{2}}\right)$ is an interpolation pair.

\section{Some Open Problems}

We will discuss four problems on wavelets that we have investigated from an operator-theoretic point of view over the past ten years, together with some related problems. The set of orthonormal dyadic one-dimensional wavelets is a set of vectors in the unit sphere of a Hilbert space $H=L^{2}(\mathbb{R})$. It is natural to ask what are the topological properties of $\mathcal{W}(D, T)$ as a subset of the metric space $H$ ? This type of question is interesting from a pure mathematical point of view, speaking as an operator theorist, and it just may have some practical consequences depending on the nature and the degree of depth of solutions.

Problem 1: [Connectedness] This was the first global problem in wavelets that we considered from an operator-theoretic point of view. In [5] we posed a number of open problems in the context of the memoir. The first problem we discussed was Problem A (in [5]) which conjectured that $\mathcal{W}(D, T)$ is norm-arcwise-connected. It turned out that this conjecture was also formulated independently by Guido Weiss and his group (see [9], [17, [26]) from a harmonic analysis point of view (our point of view was purely functional analysis), and this problem (and related problems) was the primary stimulation for the creation of the WUTAM CONSORTIUM - a team of 14 researchers based at Washington University and Texas A\&M University. (See [26], for the first publication of this group.) This connectedness conjecture was answered yes in [26] for the special case of the family of dyadic orthonormal MRA wavelets in $L^{2}(\mathbb{R})$, but still remains open for the family of arbitrary dyadic orthonormal wavelets in $L^{2}(\mathbb{R})$, as well as for the family of orthonormal wavelets for any fixed $n$ and any dilation matrix in $\mathbb{R}^{n}$. A natural related problem which also remains open, is whether the set of Riesz wavelets is connected. An intermediate problem, which is also open, asks whether given two orthonormal wavelets is there a continuous path connecting them consisting of Riesz wavelets? (Some evidence for a positive answer to this problem is given by Proposition 6.1 below, which easily shows that every point on the convex path connecting two wavelets (i.e. $(1-t) \psi+t \eta)$ is a Riesz wavelet except for perhaps the midpoint corresponding 
to $t=0.5$. Thus this problem has an easy positive solution for many pairs of orthonormal wavelets, but no way has been found to get around the midpoint obstruction to show that all pairs are connected, perhaps by some exotic type of path.) A subproblem is the same problem but for the set of frame-wavelets $\mathcal{F}(D, T)$ (now widely called framelets). Is the set of all frame-wavelets connected?; or more specifically-is the set of all Parseval frame wavelets connected? The reader can easily deduce some frame versions of Proposition 6.1 using elementary spectral theory of operators which provides some quick-and-easy partial results on paths of frames. These are tantalizing, but the main problems still remain open.

All of these connectivity problems have counterparts for other unitary systems. For wavelet systems, they remain open (to our knowledge) for all dimensions $n$ and all expansive matrix dilation factors. And for other systems, in particular in [14], we showed that for a fixed choice of modulation and translation parameters (necessarily, of course, with product $\leq 1$ ) the set of Weyl-Heisenberg (or Gabor) frames is connected in this sense, and also it is norm-dense in $L^{2}(\mathbb{R})$ in the sense of Problem 2 below. Although the Weil-Heisenberg (aka Gabor) unitary systems are of a simpler operator-theoretic structure than the wavelet systems, and this permits the use of some techniques which do not work so well in the wavelet theory, even so this perhaps is another reason to think that general connectedness results are possible within the wavelet theory.

Problem 2: [Density] Is the set $\mathcal{R} \mathcal{W}(D, T)$ of all Riesz wavelets dense in the norm topology in the Hilbert space $L^{2}(\mathbb{R})$ ? This was posed as a conjecture by Larson in a talk in August 1996 in a NATO conference held in Samos, Greece. It was posed in the same spirit as the connectivity problem above, in the sense that it asks about the topological nature of $\mathcal{R} \mathcal{W}(D, T)$ as a subset of the metric space $L^{2}(\mathbb{R})$. Like the connectivity problem it is a global type of problem. A positive answer might be useful for applications if it could be given a some type of quantitative interpretation. A subproblem of this, which was discussed in several subsequent talks, is the same density problem but for the set of frame-wavelets $\mathcal{F}(D, T)$. (Of course a positive answer for wavelets would imply it for framelets.) Like the connectivity problem, this problem makes sense for the family of Riesz wavelets for any fixed $n$ and any dilation matrix in $\mathbb{R}^{n}$.

One of the reasons for thinking that this conjecture may be positive is the following result, which we think is the most elementary application of operatortheoretic interpolation. It is abstracted from Chapter 1 of [5], although the form in [5] is a bit different.

Proposition 6.1. Let $\mathcal{U}$ be a unitary system on a Hilbert space $H$. If $\psi_{1}$ and $\psi_{2}$ are in $\mathcal{W}(\mathcal{U})$, then

$$
\psi_{1}+\lambda \psi_{2} \in \mathcal{R} \mathcal{W}(\mathcal{U})
$$

for all complex scalars $\lambda$ with $|\lambda| \neq 1$. More generally, if $\psi_{1}$ and $\psi_{2}$ are in $\mathcal{R} \mathcal{W}(\mathcal{U})$ then there are positive constants $b>a>0$ such that $\psi_{1}+\lambda \psi_{2} \in \mathcal{R} \mathcal{W}(\mathcal{U})$ for all $\lambda \in \mathbb{C}$ with either $|\lambda|<a$ or with $|\lambda|>b$. 
Proof. If $\psi_{1}, \psi_{2} \in \mathcal{W}(\mathcal{U})$, let $V$ be the unique unitary in $\mathcal{C}_{\psi_{2}}(\mathcal{U})$ given by Proposition 3.1 such that $V \psi_{2}=\psi_{1}$. Then

$$
\psi_{1}+\lambda \psi_{2}=(V+\lambda I) \psi_{2} .
$$

Since $V$ is unitary, $(V+\lambda I)$ is an invertible element of $\mathcal{C}_{\psi_{2}}(\mathcal{U})$ if $|\lambda| \neq 1$, so the first conclusion follows from Proposition 3.2. Now assume $\psi_{1}, \psi_{2} \in \mathcal{R} \mathcal{W}(\mathcal{U})$. Let $A$ be the unique invertible element of $\mathcal{C}_{\psi_{2}}(\mathcal{U})$ such that $A \psi_{2}=\psi_{1}$, and write $\psi_{1}+\lambda \psi_{2}=(A+\lambda I) \psi_{2}$. Since $A$ is bounded and invertible there are $b>a>0$ such that

$$
\sigma(A) \subseteq\{z \in \mathbb{C}: a<|z|<b\}
$$

where $\sigma(A)$ denotes the spectrum of $A$, and the same argument applies.

The above proposition indicates that Riesz wavelets are plentiful. As mentioned above, by writing $(1-t) \psi_{2}+t \psi_{1}=((1-t) V+t I) \psi_{1}$, and using the fact that the local commutant is a linear space so contains $(1-t) V+t I$, it follows that a convex combination of orthonormal wavelets is a Riesz wavelet except possibly for the mid-point corresponding to $t=0.5$. So, if $\psi$ and $\eta$ are orthonormal wavelets, the line in the vector space $L^{2}(\mathbb{R})$ containing the pair $\psi, \eta$ is in the norm closure of the set of Riesz wavelets. Operator theoretic interpolation shows that more general linear combinations of finite families of Riesz wavelets are very often Riesz wavelets. And if one considers a finite family of wavelet sets $\mathcal{C}$, with union $\mathcal{S}$, then the restricted sets of Riesz wavelets, or orthonormal wavelets, or Parseval framelets, or framelets, which are restricted in the sense that they have their frequency support contained in $\mathcal{S}$, is always connected if the family of wavelet sets is an interpolation family, and these restricted sets of wavelets are very often connected even if the interpolation family criterion fails for $\mathcal{C}$. (In fact, it is a conjecture that these restricted sets of wavelets are always connected.) Moreover, these restricted sets are dense in $L^{2}(S)$ (considered as a subspace of $L^{2}(\mathbb{R})$ ) if the family $\mathcal{C}$ is an interpolation family, and it is yet another conjecture that they are always dense in $L^{2}(S)$. It is also known (see [5] for instance) that the linear span $\mathcal{W}(D, T)$ is dense in $L^{2}(\mathbb{R})$. So these facts together suggest that it is probably true that the set of Riesz wavelets $\mathcal{R} \mathcal{W}(D, T)$ is dense in $L^{2}(\mathbb{R})$. However, the problem remains open. Also, as mentioned above under the connectivity problem, one can easily deduce some frame versions of Proposition 6.1 using elementary spectral theory of operators which provides some quick-and-easy partial results on density of Riesz wavelets and framelets. However, the general problem for Riesz wavelets remains open. (For the density problem for framelets, we mention that Marcin Bownick has recently obtained a significant positive result! It appears that he has solved the problem positively for framelets. But apparently it remains open for Riesz wavelets.)

As with the connectedness problem, all of these problems have counterparts for other unitary systems. For wavelet systems, they remain open (to our knowledge) for all dimensions $n$ and all expansive matrix dilation factors (except for the 
recent interesting framelet density result of Bownick mentioned above). And for other systems, as mentioned in the context of Problem 1, in [14] we showed that for a fixed choice of modulation and translation parameters with product $\leq 1$ the set of Weyl-Heisenberg (or Gabor) frames is dense in $L^{2}(\mathbb{R})$ in this sense. This is another reason to think that general density results might be possible within the wavelet theory.

Problem 3: [Frequency Support] (See section 4.4.) [Must the support of the Fourier transform of a wavelet contain a wavelet set?] This conjecture was posed about 10 years ago, by Larson, and the problem still remains open for the case of dimension 1 and dilation factor 2. It makes sense for any finite dimension $n$ and any matrix dilation, and it apparently remains unsolved in any case. It has been studied by several researchers, and Z. Rzeszotnik, in particular, has made some progress on the problem. A related problem, also posed by Larson, (see section 4.4) asks whether a wavelet which has minimal support in the frequency domain is necessarily an MSF wavelet. (In other words: Is a minimal support set in the frequency domain necessarily a wavelet set).

Problem 4: [Normalization] (See section 3.3.1.) If $\{\psi, \eta\}$ is a pair of dyadic orthonormal wavelets, does the interpolation unitary $V_{\psi}^{\eta}$ normalize $\{D, T\}^{\prime}$ ? As mentioned above, the answer is yes if $\psi$ and $\eta$ are $s$-elementary wavelets. This problem makes sense for orthonormal wavelets in higher dimensions for matrix dilation factors, and for other scalar dilations in one dimension. We know of no counterexample for any of these cases. However, the problem might just lie in the fact that in most cases, other than for wavelet sets, we have no reasonable techniques for doing the computations. This problem was also discussed in context in Section 3.3.1, and it could be the most important problem remaining in the direction of further development of the unitary system approach to wavelet theory.

\section{References}

[1] E. A. Azoff, E. J. Ionascu, D. R. Larson, and C. M. Pearcy, Direct Paths of Wavelets. Houston J. Math. 29 (2003), no. 3, 737-756.

[2] L. Baggett, H. Medina, and K. Merrill, Generalized Multi-resolution Analyses and a Construction Procedure for all Wavelet Sets in $R^{n}$. J. Fourier Anal. Appl. 5 (1999).

[3] J. J. Benedetto and M. Leon, The Construction of Single Wavelets in D-dimensions. J. Geom. Anal. 11 (2001), no. 1, 1-15.

[4] X. Dai, Y. Diao, Q. Gu and D. Han, Wavelets with Frame Multiresolution Analysis. J. Fourier Analysis and Applications 9 (2003), 39-48.

[5] X. Dai and D. Larson, Wandering Vectors for Unitary Systems and Orthogonal Wavelets. Mem. Amer. Math. Soc. 134 (1998).

[6] X. Dai, D. Larson and D. Speegle, Wavelet Sets in $R^{n}$. J. Fourier Anal. Appl. 3 (1997), no. 4, 451-456.

[7] X. Dai, D. Larson, and D. Speegle, Wavelet Sets in $R^{n}-$ II. Contemp. Math 216 (1998), 15-40. 
[8] I. Daubechies, Ten Lectures on Wavelets. SIAM Philadelphia, PA, 1992.

[9] X. Fang and X. Wang, Construction of Minimally-supported Frequencies Wavelets. J. Fourier Anal. Appl. 2 (1996), 315-327.

[10] T. N. T. Goodman, S. L. Lee and W. S. Tang, Wavelets in Wandering Subspaces. Transactions AMS 338 (1993), 639-654.

[11] Q. Gu, On Interpolation Families of Wavelet Sets. Proc. Amer. Math. Soc. 128 (2000), 2973-2979.

[12] D. Han, Wandering Vectors for Irrational Rotation Unitary Systems. Transactions AMS 350 (1998), 309-320.

[13] D. Han, J-P. Gabardo, and D. R. Larson, Gabor Frames and Operator Algebras. Wavelet Appl. in Signal and Image Proc., Proc. SPIE 4119 (2000), 337-345.

[14] D. Han and D. R. Larson, Frames, Bases and Group Representations. Memoirs AMS 697 (2000).

[15] D. Han and D. R. Larson, Wandering Vector Multipliers for Unitary Groups. Transactions AMS 353 (2001), 3347-3370.

[16] C. Heil, P. E. T. Jorgensen, and D. R. Larson (eds.), Wavelets, Frames and Operator Theory, Contemp. Math., 345. AMS, Providence, RI, 2004, College Park, MD, Jan. 15-21, 2003.

[17] E. Hernandez, X. Wang and G. Weiss, Smoothing Minimally Supported Frequency (MSF) Wavelets: Part I. J. Four. Anal. Appl. 2 (1996), 329-340.

[18] E. Ionascu, D. Larson, and C. Pearcy, On the Unitary Systems Affiliated with Orthonormal Wavelet Theory in n-dimensions. J. Funct. Anal. 157 (1998), no. 2, 413-431.

[19] R. Kadison and J. Ringrose, Fundamentals of the Theory of Operator Algebras, Vol. $I$ and II. Academic Press, Inc. 1983 and 1985.

[20] K. Kornelson and D. Larson, Rank-one Decomposition of Operators and Construction of Frames. Wavelets, Frames, and Operator Theory, Contemp. Math., 345. AMS, 2004, pp. 203-214.

[21] D. R. Larson, Von Neumann Algebras and Wavelets. Operator Algebras and Applications (Samos, 1996), 267-312. NATO Adv. Sci. Inst. Ser. C Math. Phys. Sci., 495, Kluwer Acad. Publ., Dordrecht, 1997.

$[22]$ D. R. Larson, Frames and Wavelets from an Operator-theoretic Point of View, Operator Algebras and Operator Theory (Shanghai, 1997), 201-218. Contemp. Math., 228, AMS, Providence, RI, 1998.

[23] D. R. Larson, E. Schulz, D. Speegle, and K. Taylor, Explicit Cross Sections of Singly Generated Group Actions, to appear.

[24] D. R. Larson, W. S. Tang, and E. Weber, Multiwavelets Associated with Countable Groups of Unitary Operators in Hilbert Spaces. Int. J. Pure Appl. Math. 6 (2003), no. 2, 123-144.

[25] D. Speegle, The s-elementary Wavelets are Path-connected. Proc. Amer. Math. Soc. 132 (2004), 2567-2575

[26] Wutam Consortium, Basic Properties of Wavelets. J. Four. Anal. Appl. 4 (1998), 575-594. 
David R. Larson

Department of Mathematics

Texas A\&M University

College Station, TX 77843-3368, U.S.A.

e-mail: larson@math.tamu.edu 